\title{
Surface and Quantum Effects in Nanosized Semiconductor
}

\author{
Augustine Ike Onyia, Henry Ifeanyi Ikeri, Abraham Iheanyichukwu Chima
}

Department of Industrial Physics, Enugu State University of Science and Technology, Enugu, Nigeria

\author{
Email address: \\ ifeanyihenry75@yahoo.com (H. I. Ikeri)
}

\section{To cite this article:}

Augustine Ike Onyia, Henry Ifeanyi Ikeri, Abraham Iheanyichukwu Chima. Surface and Quantum Effects in Nanosized Semiconductor. American Journal of Nano Research and Applications. Vol. 8, No. 3, 2020, pp. 35-41. doi: 10.11648/j.nano.20200803.11

Received: July 29, 2020; Accepted: August 11, 2020; Published: August 19, 2020

\begin{abstract}
Novel properties of nano-scale semiconductors based on the surface and quantum effects have been studied and applications identified. Spherical potential well model is used to study quantum effect whereas basic geometrical models are used for the surface effect. We have shown such effects to be the fundamental factors responsible for the novel nanosized semiconductor characteristics different from the bulk of same material. It is found that the surface area to volume ratio follows inverse power law. Thus at nanoscale, the surface to volume ratio increases significantly to enhance chemical reactivity. In addition, the increased surface area makes most nananocrystals highly soluble in liquid and dramatically lowers their melting temperature. The result strongly suggests also that the shape of the nanoparticles influences the surface area which has huge impact on their properties and performance. Our results of quantum size effect reveal that spatial confinement of charge carriers within semiconductor nanocrystals significantly modulates their properties such as size dependent absorption and emission spectra with non-zero discrete electronic transition energies as well as their blue shift band gaps. Thus by changing the size of the particle, we can literally fine-tune a material property of interest such as optical, electrical, and surface area. Specifically we found that InAs and InSb nano semiconductor optical absorption spectrum, in contrast to their bulk, can be tuned in broad range of UV to IR regions which are favorable operating wavelengths for nano photonic technology such as IR photo detectors and full spectrum solar cells applications.
\end{abstract}

Keywords: Nanocrystal, Quantum Confinement, Quantum Effect, Semiconductor, Surface Effect

\section{Introduction}

The large surface area, discrete electronic transitions and tunable chemical, biological and physical properties of semiconductor nanocrystals have, in recent times, attracted research interests. Microelectronics have advanced towards nanoscale electronics such that microelectronics basic building blocks: molecules, atoms, and even electrons, can be manipulated. The physical laws which govern the behavior of particles in nano-scale regime are significantly different from the laws of classical physics which control the operation of macro and microelectronic devices [1]. Quantum mechanical model is better used to describe motion and energy of the system in this small length scale dimension $[2,3]$.

A potent example of nanoscopic phenomenon is quantum effect tuning of the material properties. Precisely, as the size of semiconductors are reduced to nanoscale, the properties become size dependent contrary to the bulk in which the properties of matter are related to the atoms and molecules that constitute the substance and their chemical bonds [4]. A second prime example is evident in quantum tunneling in which if a particle with energy $E$ encounters a barrier of energy $U$ the probability of finding the particle across the barrier if $\mathrm{E}<\mathrm{U}$ is not zero but finite [5]. In addition nanoparticles exhibit wave-like behaviour known as matter wave introduced by de Broglie, with a wavelength that is inversely dependent on the particle's linear momentum. The gravitational forces become negligible due to the small mass in this size range whereas electromagnetic forces become increasingly more important and are dominant in determining the behavior of atoms and molecules [6].

Nanosized semiconductors exhibit quantum structures, one in which the motion of the charge carrier is confined in one or more directions by potential barriers, such as in quantum wells, quantum wires, and quantum dots in contrast to bulk semiconductors that have their charge carriers free to move within their respective bands in all the three directions, [7]. When the charge carriers are confined in one-direction, the 
energy is only continuous in the two-dimensional space, we have quantum wells or quantum films. Also, if the carriers are confined in the two-directions and the energy is continuous in one dimension, we have quantum wires or quantum rods. In the same way if the charge carriers are confined in all three spatial directions and there is zero dimensional degree of freedom, the resulting material is termed quantum dots (QDs) [8].

Among the family of the confined structures, semiconductors QDs have emerged as the ultimate limit in charge carrier confinement due to the 3D space carrier confinement. In such structures, energy bands display discrete electronic states reminiscent of isolated atoms and electrical and optical properties change significantly from those of bulk materials [9] and the fundamental band gap of the system becomes size-dependent contrary to the bulk in which band gap maintains its original value due to the continuous energy state. QD band gap is thus tunable [10]. Thus by changing the size of the particle we can literally fine-tune material properties thereby allowing the engineering of new materials that have their bandgaps at custom designed energies to suite device applications [11].

The surface area of the semiconductor nanocrystal also plays a key role on the properties of the material. The nanocrystals have a relative larger surface area when compared to the same volume of material made up of bigger particles [12]. The increased surface area presents more atoms for increased chemical and physical activity such as catalysis and detection reactions [13-15].

In addition, increased surface area has the following effect: (i) it increases the surface energy and hence decreases the melting point:- $3 \mathrm{~nm}$ CdSe nanosized semiconductor melts at $700 \mathrm{~K}$ whereas the bulk counterpart melts at $1678 \mathrm{~K}$ (ii) it makes most nano semiconductors highly soluble in liquid which found applications in pigments, paints, medicine pills etc (iii) it increases the adsorption processes which is useful in applications like water purification device, waste recovery techniques, manufacturing of perfumes and kadhesive among others [16]. Other researchers have shown that the shape of the nanoparticles influences the surface area which may have huge effect on their properties.

Many benefits of semiconductor depend on the fact that it is possible to tailor the material structures to extremely small scales to achieve specific properties. Using quantum or surface effect, materials can effectively be made more reactive, better electrical conductors, stronger, lighter or more durable. The size effect and surface effect are the two fundamental effects that modulate the behavior of semiconductors in nano size regime. The unique physical and chemical properties of semiconductor nanocrystals make them suitable for applications in emerging technologies, such as catalysis, nanoelectronics, nanophotonics, energy conversion, non-linear optics, solar cells, detectors etc [17]. In this paper we have provided a comprehensive theoretical study of the impact of surface and quantum effects on the physical and chemical properties and applications of semiconductor nanocrystals.

\section{Fundamental Concept}

The properties of semiconductors vary as their size approaches the nanoscale dimension and as the greater proportion of atoms at the surface of the material becomes significant leading to dramatic change in the physical and chemical properties of the material contrary to their equivalent chemical compound in large dimensions making the properties of nanoparticles strongly size-dependent. These novel properties stems from the quantum and surface effects that matter exhibits at the nanoscale, which serves as fundamental tools to push the basic scientific and engineering knowledge in many area of modern science and technology enabling novel applications and interesting materials.

In a bulk semiconductor, the valence and conduction bands are close and it is easy to excite an electron into the conduction band where they can move around. Once the electron is excited into conduction band it leaves an empty space behind in the valence band called a hole as shown in Figure 1. The negatively charge electron and positively charged hole interact through the electrostatic Coulomb potential to form a bound state known as exciton.

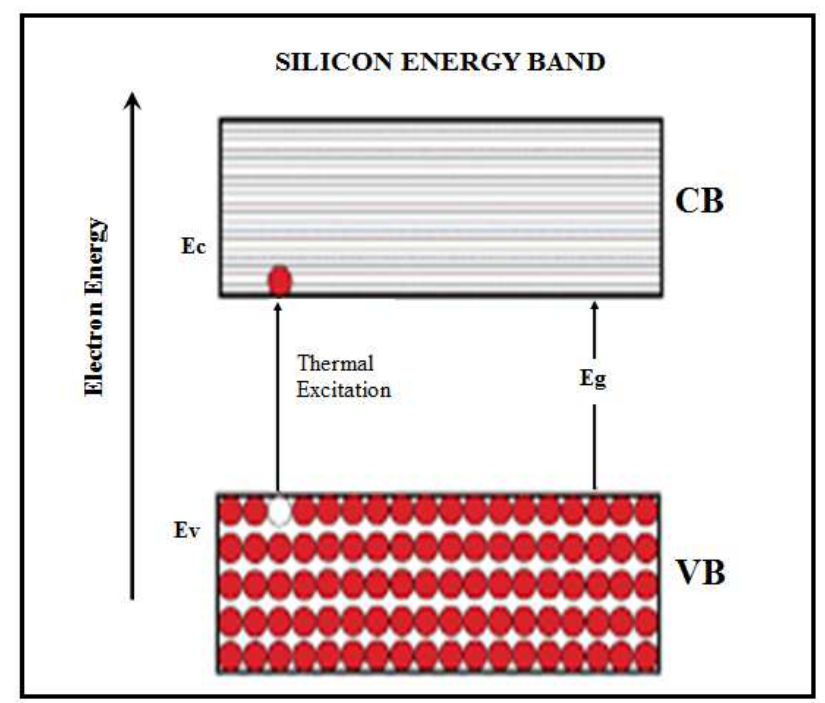

Figure 1. Semiconductor energy band diagram.

The exciton behaves like a hydrogen atom in which a hole instead of the proton forms the nucleus. The average distance between the excited electron in the conduction band and hole it leaves behind in the valence band is defined as the exciton Bohr radius $r_{B}$ mathematically expressed as (18):

$$
r_{B}=\frac{h^{2} \varepsilon}{4 e^{2} \pi^{2}}\left(\frac{1}{m_{e}^{*}}+\frac{1}{m_{h}^{*}}\right)
$$

where $h, \varepsilon, e, m_{e}^{*}$ and $m_{h}^{*}$ are plank constant, dielectric constant, electronic charge, effective masses of electron and hole respectively. A central concept in quantum physics is the wave-particle duality, introduced by de Broglie, in which a nanoparticle can be associated with a matter wave with a wavelength $\lambda_{B}$ known as de Broglie wavelength expressed in terms of the particle linear momentum $\mathrm{p}$ and Planck constant $\mathrm{h}$ as: 


$$
\lambda_{B}=\frac{h}{p}
$$

The exciton Bohrs radius and de Broglie wavelength are characteristic length scales that delineate the transition between the bulk state and nanoscale regime upon which the quantum effects are used to describe the particles behaviour. Thus whenever the dimension of semiconductor materials is reduced to a size comparable with de Broglie wavelength or exciton's Bohr radius they become small enough to confine their charge carriers and produce quantum effects [19], and the size-dependent properties become apparent resulting to novel properties different from their bulk values. The quantum effect describes the physics of material properties in nanoscale regime. This effect is not significant going from macro to micro dimensions. Its only becomes dominant when the nanometer size range is reached. Thus materials reduced to the nanoscale can suddenly display very different properties compared to what they exhibit on a macroscale which represents a significant step forward.

The energy levels of the semiconductor nanocrytals can be understood by solving Schrodinger equation of quantum mechanics for the electrons and holes in the potential wells located by the band discontinuities. This is a real life scenario of the famous particle in a box, whose energy level is given as [20]:

$$
E n=\frac{n^{2} h^{2}}{8 m L^{2}}
$$

This energy term represents the infinite potential well contribution due to confinement of the charge carriers. Here $\mathrm{L}$ is the length of the confinement region, $\mathrm{m}$ is the mass of the particle, $\mathrm{h}$ is the planck constant and $\mathrm{n}$ is the quantum number that labels the different confined energy levels of the particle in the well. In general, charge carriers (electrons and holes) in a semiconductor nanocrystal are confined in all spatial 3D [21]. So we considered these charge carriers trapped in an infinite 3-dimensional potential well. The simplest shape of a three-dimensional box considered is sphere. We therefore extend the concept of energy in a onedimensional potential well to the case of a sphere of interest applying the necessary boundary conditions and obtained the lowest allowed energy level (kinetic energy) as:

$$
E_{n, m}=\frac{\beta_{n, m}^{2}}{8 m R^{2}} h^{2}
$$

where:

1) $\beta_{n, m}^{2}$ is the mth root of the spherical Bessel function of quantum number $n$

2) $R$ is the radius of the QD

3) $\mu$ is the reduced effective mass

4) $E_{n, m}$ is the confinement energy (energy state) of the electron and hole.

The ground state kinetic energy which is the commonly observed state corresponds to $\left(\beta_{1,0}^{2}=\pi\right.$ and $\left.n=1\right)$ and is given as:

$$
E_{1,0}=\frac{h^{2}}{8 m R^{2}}
$$

This energy term represents the infinite potential spherical well contribution due to quantum confinement of charge carriers in $3 \mathrm{D}$. While the above equation is for one particle in a quantum sphere, we extend the same concept to the two particle exciton (electron and a hole) and obtained:

$$
E=\frac{h^{2}}{8 R^{2}}\left(\frac{1}{m_{e}^{*}}+\frac{1}{m_{h}^{*}}\right)
$$

This energy term is derived from the particle-in-aspherical box solution of the Schrödinger equation that has been expanded to a sphere with an electron particle and a corresponding hole, where $m_{e}^{*}$ and $m_{h}^{*}$ are the effective masses of the electrons and holes, respectively. Columbic interaction between the negatively (electron) and positively (hole) charged each with charge of magnitude e according to Coulomb's law, is on the order of:

$$
E=\frac{-1.8 e^{2}}{4 \pi \varepsilon_{0} \varepsilon_{r} R}
$$

where; 1.8 is a coefficient due to wave function overlap between the electron and hole in the first excited state whereas the minus sign indicates that two particles (electron and hole) are of opposite charges. This energy third term represents the electrostatic energy contribution due to coulomb's attraction between the electron and the hole. The last energy term contribution is the infinite crystal bandgap energy $E_{g_{(b u l k)}}$ of the host semiconductor which is a characteristic of the material. The ground state excitation energy of semiconductor nanocrystals is the sum of the three energy contributions thus given as:

$$
E_{g\left(Q D_{S}\right)}=E_{g_{(\text {bulk })}}+\frac{h^{2}}{8 R^{2}}\left(\frac{1}{m_{e}^{*}}+\frac{1}{m_{h}^{*}}\right)-\frac{1.8 e^{2}}{4 \pi \varepsilon_{0} \varepsilon_{r} R}
$$

The fundamental relationship between the excitation energy $E_{g\left(Q D_{s}\right)}$ and the optical absorption wavelength $\lambda_{\left(Q D_{S}\right)}$ of semiconductor nanocrystals is expressed as:

$$
\lambda_{\left(Q D_{S}\right)}=\frac{h C}{E_{g\left(Q D_{S}\right)}}
$$

The other crucial aspect of nanocrystals responsible for their novel behaviour is the surface effect. Thus surface area to volume ratio for a material or substance made of nanoparticles has a significant effect on the properties of the material. Nanoscale materials have a relatively larger surface area when compared to the same mass of material in bulk form [22]. This makes nanocrystals more chemically reactive (in some cases materials that are inert in bulk form are reactive in their nanoscale form), which has significant impact on their strength or electrical properties [23]. We considered spherical and cubic nanocrystals geometries and applied basic geometric formulas to establish a fundamental surface area to volume relationship. For spherical shape semiconductor nanocrystal the Equation for the surface area $\mathrm{S}$ of the sphere of radius $r$ is expressed as:

$$
S=4 \pi r^{2}
$$

Volume of the sphere $\mathrm{V}$ is defined as: 


$$
V=\frac{4}{3} \pi r^{3}
$$

Then the surface area to the volume ratio $\alpha$ becomes:

$$
\alpha=\frac{4 \pi r^{2}}{\frac{4}{3} \pi r^{3}}
$$

Thus

$$
\begin{gathered}
\alpha=\frac{3}{r} \\
\rightarrow \alpha \propto \frac{1}{r}
\end{gathered}
$$

Similarly for cubic shape semiconductor nanocrystal of the length of a side $\mathrm{L}$ the Equation. of the surface area $\mathrm{S}$ of the sphere is:

$$
S=6 L^{2}
$$

Volume of the cube $\mathrm{V}$ is:

$$
V=L^{3}
$$

Then the surface area to the volume ratio $\beta$ becomes:

$$
\beta=\frac{6 L^{2}}{L^{3}}
$$

Thus

$$
\begin{aligned}
& \beta=\frac{6}{L} \\
& \rightarrow \beta \propto \frac{1}{L}
\end{aligned}
$$

The radius $r$ and length $L$ are both referred here as sizes hence surface to volume ratio for both spherical and cubic geometrical nanocrystals indicates that the surface area increases faster relative to the volume as crystal size decreases Maximum exposed surface area signifies an improved reactivity thus greater percentage of the material atoms are exposed for potential reaction.

In nanoscience the shape of the nanoparticles also plays critical role on the surface area of the material and influences their properties. Given Equ. 10 the surface area per unit volume for a sphere $\alpha=\frac{3}{r}$ and Equ. 14 for the cubic shape, $\beta=\frac{6}{L}$, therefore between a spheric and cubic shapes of same volume there will be larger increased surface area in the cube relative to the sphere and for this particular reason not only the size of the nanoparticles is vital but also their shape.

\section{Materials and Methods}

The verified experimental parameters we used for the this study are: effective masses of electron $m_{e}^{*}$, and hole, $m_{h}^{*}$, dielectric constants and bandgap energy $E g$ for $\mathrm{GaAs}, \mathrm{InSb}$ and InAs QDs as shown in the Table 1 whereas the constant parameters used in the computation are listed in the appendix. Variation in excitonic kinetic energy, excitonic excitation energy and optical wavelength as a function of nanocrystal size was computed using Equation 3, 6 and 7 respectively, in order to ascertain the novel properties of semiconductors in nanoscale regime. The input parameters used in the theoretical calculation have been listed in Table 1.' We have also used the Equations 11 and 14 for the computation of surface area per unit volume of various sizes of the nanocrystal for the spherical and cubic nano semiconductors respectively

Table 1. The material parameters used for the Study.

\begin{tabular}{lllll}
\hline QDs & $\boldsymbol{E} \boldsymbol{g}$ & $\boldsymbol{m}_{\boldsymbol{e}}^{*}$ & $\boldsymbol{m}_{\boldsymbol{h}}^{*}$ & $\boldsymbol{\varepsilon}_{\boldsymbol{r}}$ \\
\hline InAs & $0.36 \mathrm{eV}$ & $0.02 \mathrm{~m}_{\mathrm{o}}$ & $0.40 \mathrm{~m}_{\mathrm{o}}$ & 15.15 \\
InSb & $0.17 \mathrm{eV}$ & $0.02 \mathrm{~m}_{\mathrm{o}}$ & $0.40 \mathrm{~m}_{\mathrm{o}}$ & 16.80 \\
$\mathrm{GaAs}$ & $1.42 \mathrm{eV}$ & $0.06 \mathrm{~m}_{\mathrm{o}}$ & $0.51 \mathrm{~m}_{\mathrm{o}}$ & 12.90 \\
\hline
\end{tabular}

\section{Result and Discussion}

Figure 2 shows the excitonic kinetic energy as a function of semiconductor nanocrystal size. Thus as the confinement length is reduced charge carriers assume higher kinetic energies resulting in increased energy levels. As the kinetic energy is inversely dependent on the effective mass this implies that lighter particles experience stronger effect which in turn means that light and heavy hole states possess different energies in contrast to parental bulk. The spatial confinement of charge carriers within the quantum box has two fundamental consequences that significantly modulate the carrier states. Firstly, it causes the electronic states to become discrete with controllable atomic-like states which shift the effective band edge to higher energies in sharp contrast to the bulk whose electronic energy levels are distributed in continuous bands. Thus energy level spacing increases sharply with decreasing nanocrystal size. Secondly, confinement brings electrons and hole closer and therefore enhances the radiative recombination likelihood compared to the bulk where electron and hole are not spatially confined and can be easily dissociated enhancing the probability of non radiative recombination.

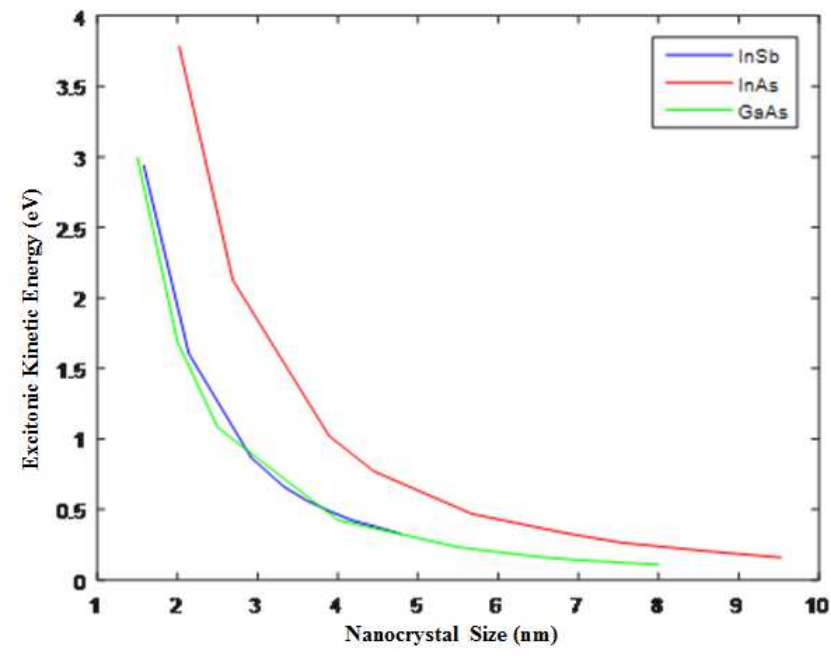

Figure 2. Excitonic kinetic energy versus nanocrystal size for InSb, InAs and GaAs QDs.

As the dot radius increases, the kinetic energy for all nanocrystal decrease exponentially but never reaches zero. It means that charge carriers in the nanocrystals can never be in 
a state of rest and even in the ground state the charge carriers possess a non-zero minimum kinetic energy.

In the Figure 3 showing the excitation energy as a function of nanocrystal size, a substantial increase in the exciton excitation energy and hence the separation between the valence and conduction bands with decreasing crystal size is observed. The increase of excitation energy is due to quantum confinement. This therefore implies that fundamental bandgap energy of a nanoscale semiconductor is size-dependent and can be tuned by varying the crystallite size. Accordingly, a large optical blue shift is observed in the excitation energy spectrum as compared with the bulk materials that are characterized by composition-dependent bandgap energy. As earlier stated nanosize semiconductors have quantized energy levels and as a result electron transfer can only occur between these discrete electronic states. Consequently only light of certain dimension can be absorbed leading to monochromatic emissions. The structures with such a discrete energy spectrum are the basis for the development of new types of nanoelectronic devices. The tunable excitation energy allows nanosize semiconductors to be optimally engineered for the different applications particularly in solar cells in which they can be tuned to harvest the full broad spectrum of solar radiation.

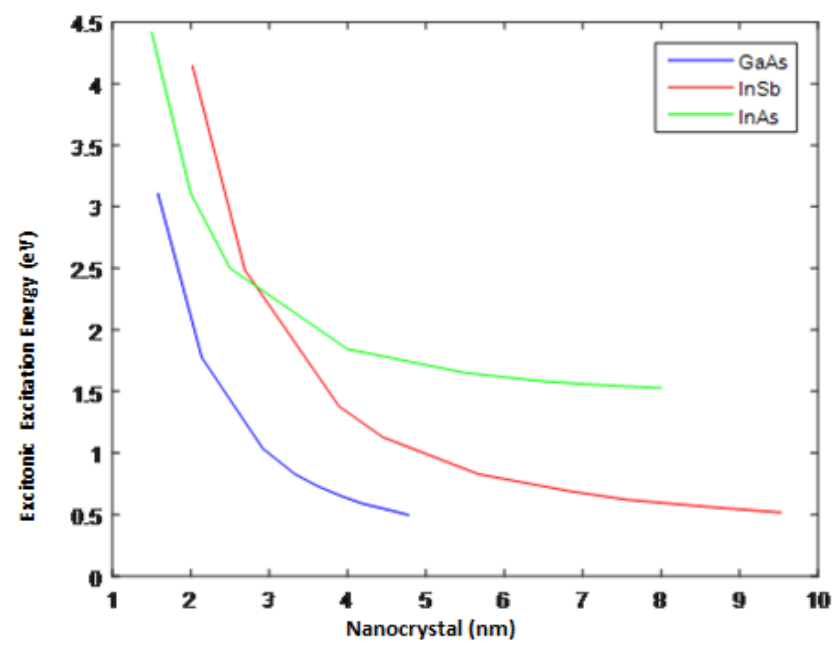

Figure 3. Excitonic excitation energy vrsus nanocrystal size for InSb, InAs and GaAs QDs.

The Figure 4 shows semiconductor nanocrystal size as a function of optical wavelength. The absorbance wavelength shows positve correlation with the crystalline size. As the cryatal decreases in size absorption edge shift towards shorter wavelengths (blue shift) which demonstrate size dependent absorption and fluorescence spectra. Thus tuning the size of semiconductor nanocrystal means tuning the wavelengths of absorption and fluorescence by the nanocrystal, which in turn means same material emits different colours at various crystallite size and are being studied as alternative light emitting sources. By varying GaAs, InSb and InAs QDs in broad range, wavelengths covering the whole of Ultraviolet UV to visible spectra were observed. However, increasing the radius further for InAs and $\mathrm{InSb}$, wavelength covering
$700 \mathrm{~nm}$ to $2500 \mathrm{~nm}$ can be absorbed indicating excellent absorption wavelengths in UV to infrared IR region. As a result, their light absorption can be tuned throughout the UV, visible and IR ranges which are favorable operating wavelengths for photonic technology such as IR photo detectors and solar cells applications.

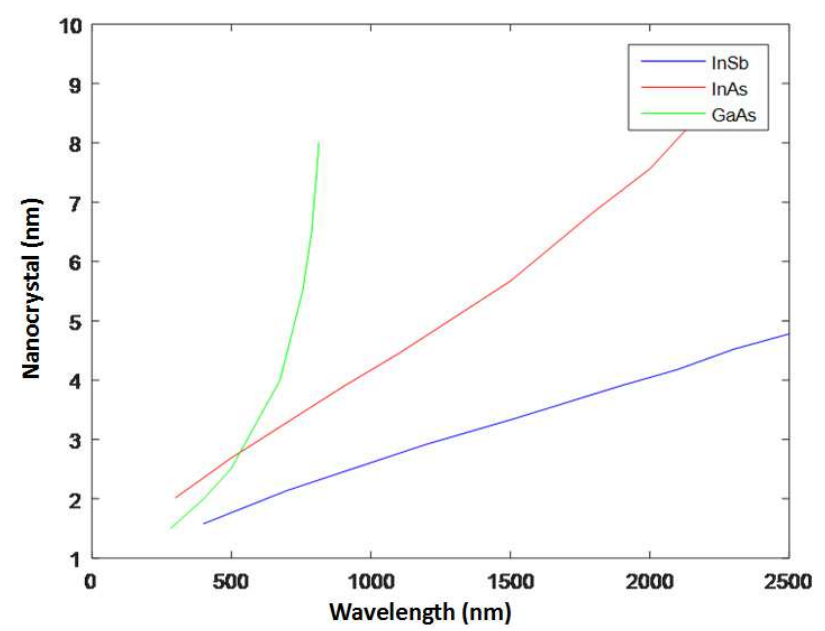

Figure 4. Nanocrystal size versus wavelength for InSb, InAs and GaAs QDs.

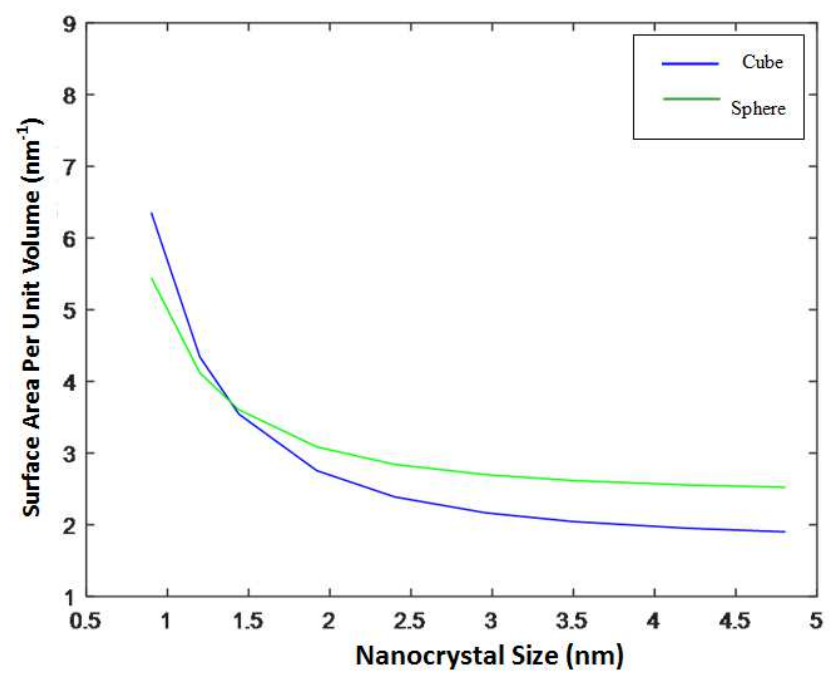

Figure 5. Surface area per unit volume versus spherical and cubic nanocrytal size.

The Figure 5 shows surface area per unit volume for different crystal sizes both for the cubic and spherical shape nanocrystals. It is found that the surface area to volume ratio scales as inverse power law and increases as the size of the particle decreases. This also implies for a given volume of material made up of smaller particles, the surface area of the material increases and thus has a relative larger surface area when compared to the same volume of material made up of larger particles. Generally, the higher the surface area implies higher surface atoms. Thus greater portion of their existing atoms are found at the surface. This leads to nanoscale semiconductors being more chemically reactive. As chemical reactions occur between particles that are on the surface, a given mass of nanomaterial will be much more reactive than 
the same mass of material made up of large particles. In general perspective physical and chemical properties of a material greatly depend on the surface atoms, and the fact that a significant number of atoms exist at the surface in nanocrystals implies tremendous effects on the reactions that occur at interface (surface) such as catalysis and detection reactions. The active site in the catalyst where the reaction takes place is significantly favored by the surface effect and thus increases with the decreased in the size of the nanocatalysts. This dramatically enhances the ultimate goal of catalysis processes to increase the rate of a given reaction.

Nanomaterias also play critical role in detection applications like biosensors or chemical sensors for detection of a specific biological and chemical compound within a mixture. The detection reaction like catalysis also occurs at the material surface. The increased surface to volume ratio as matter is reduced to nanoscales increases the surface area available for detection. This strongly indicates that further reduction in the crystal size creates more detection sites thereby enabling multiple detection of analytes. In this way it is possible to engineer super small multiplex detection device that can test and detect more than one analyte at a time. Further consequence of the increased surface area on nanoparticles is that it will have tremendous effect on the melting temperature owing to the fact that surface atoms are easily ionized than the bulk atoms, thus the total energy required to surmount the intermolecular forces that hold the atoms fixed will be less which have a dramatic depression on their melting point. Furthermore, the increased surface area makes most nananocrystal highly soluble in liquid which found applications in many chemical industries. The results reveal that whenever materials are reduced to nanoscale size range the shape of the material plays critical role on the properties of the material. The comparison between the cubic and spherical nanocrystals demonstrates a higher surface area to volume ratio for cubic geometries. This strongly suggests that the shape of the matter influences the surface area and may have significant effect in the absorption and emission process of nanoparticles.

\section{Conclusions}

The ability to fabricate structures in nanometer regime has already demonstrated a potential route for next generation nanoscale optics, electronics, chemical and biomedical devices. When the size of semiconductor materials is reduced to nanoscale, their physical and chemical properties change significantly from those of larger dimension scale materials resulting to unique properties such as size dependent and non zero discrete electronic transitions and increased surface to volume ratio (large surface area). Thus electrical conductivity of the semiconductor nanocrystals and the corresponding optical properties such as absorption and fluorescence spectra, absorption coefficient and refractive index can be altered significantly by varying the crystalline sizes and the relatively larger area of nano semiconductors strongly favors surface reactions like catalysis and detection reactions.
Additionally, the increased surface to volume ratio makes most nananocrystal highly soluble in liquid and dramatically lowers their melting point, which found applications in many chemical industries.

We have also shown that properties of nanoparticles are not only determined by size but also by their shape. These unique properties are due the quantum and surface effects that matter exhibits at the nanoscale, and have shown great value for application in emerging technologies, such as nanoelectronics, nanophotonics, energy conversion, nonlinear optics, miniaturized sensors detectors and many others. However, in this work we did not account on the mathematical relationship to aid the fabrication of nanoparticles to optimize their shape for specific property. Therefore more efforts should be focus on the formulation of model that controls specific property for a given shape.

\section{Appendix}

Table 2. Approximate values of some physical constants.

\begin{tabular}{ll}
\hline Quantity & Value \\
\hline Electronic Charge (e) & $1.6 \times 10^{-19} \mathrm{C}$ \\
Speed of light $(\mathrm{c})$ & $3 \times 10^{8} \mathrm{~m} / \mathrm{s}$ \\
Planck's constant $(\mathrm{h})$ & $6.63 \times 10^{-34} \mathrm{~J} . \mathrm{S}$ \\
Electron rest mass $\left(\mathrm{m}_{0}\right)$ & $9.11 \times 10^{-34} \mathrm{~kg}$ \\
Electron volt $(\mathrm{eV})$ & $1.6 \times 10^{-19} \mathrm{~J}$ \\
Permittivity of free space & $8.85 \times 10^{-12} \mathrm{fm}^{-1}$ \\
Pi $(\pi)$ & 3.142 \\
\hline
\end{tabular}

\section{References}

[1] Bawendi MG, Steigerwald ML, Brus LE. The quantum mechanics of larger semiconductor clusters ("quantum dots"). Annu. Rev. Phys. Chem. 1990; 41: 477-496.

[2] Davies, H. (2006). "The Physics of Low Dimensional Semiconductors;" An introduction (6 ${ }^{\text {th }}$ reprint Ed.) Cambridge University Press.

[3] Warnock J, Awschalom DD. Quantum size effects in simple colored glass. Phys. Rev. B. 1985; 32: 5529-5531.

[4] Ekimov AI, Onushchenko AA. Quantum size effect in the optical-spectra of semiconductor micro-crystals. Sov. Phys. Semicond. 1982; 16: 775-778.

[5] Neil T. ON quantum tunneling in real time. New journal of Physics 2004: 16.

[6] Weinberger P. Revisiting Louis de Broglie's famous 1924 paper. Philosophical magazine letters 2006: 86.

[7] Borrelli NF, Hall DW, Holland HJ, Smith DW. Quantum confinement effects of semiconducting microcrystallitesin glass. J. Appl. Phys. 1987; 61: 5398-5409.

[8] Ikeri, H. and Onyia, A. (2017). Theoretical Investigation of Size Effect on Energy Gap of Quantum Dots Using Particle in a Box Model.

[9] Harbold, J. and Monica, P (2008). The Electronic and Optical Properties of Colloidal Lead Selenide Semiconductor Nanocrystal: Ph.D. Dissertation. Cornel University. Ithaca, New York. 
[10] Onyia, A. I. and Ikeri, H. I. (2018). Theoretical Study of Quantum Confinement Effect on Quantum Dots Using Particle in a Box Model. Journal of Ovonic Research, 14 (1): 49-54.

[11] Nirmal M, Brus L. Luminescence photophysics in semiconductor nanocrystals. Acc. Chem. Res. 1999; 32: 407 414.

[12] Gupta AK and Gupta M. 2005. Synthesis and surface engineering of iron oxide nanoparticles for biomedical applications. Biomaterials 26: 3995-4021.

[13] Falsig, H.; Hvolbaek, B.; Kristensen, I. S.; Jiang, T.; Bligaard, T.; Christensen, C. H.; Norskov, J. K. Trends in the catalytic $\mathrm{CO}$ oxidation activity of nanoparticles. Angew. Chem. Int. Ed. 2008, 47, 4835-4839.

[14] Sheehan PE, Whitman LJ. Detection limits for nanoscale biosensors. Nano Lett. 2005; 5: 803-807.

[15] Valden, M.; Lai, X.; Goodman, D. W. Onset of catalytic activity of gold clusters on titania with the appearance of nonmetallic properties. Science 1998, 281, 1647-1650.

[16] Alivisatos AP. Perspectives on the physical chemistry of semiconductor nanocrystals. J. Phys. Chem. 1996; 100: $13226-13239$.

[17] Hauck TS and Chan WC. 2008. Assessing the effect of surface chemistry on gold nanorod uptake, toxityand expression in mammalian cells. Small 4: 157-159.

[18] Chukwuocha, E., and Onyeaju, M. (2012), Effect of Quantum Confinement on the Wavelength of CdSe Quantum Dots. International Journal of Science and Technology Research, 1 (7), 21-24.

[19] Nisha, P. and Amrita, D. (2016). Theoretical study of Dependence of Wavelength on Size of Quantum Dot. Intl Journal of Scientific Research and Development, 4 (1): 126130.

[20] Brus LE. Electron-electron and electron-hole interactions in small semiconductor crystallites - the size dependence of the lowest excited electronic state. J. Chem. Phys. 1984; 80: 4403-4409.

[21] Ikeri, H and Onyia, A. (2019). The Dependence of Confinement Energy on the Size of Quantum Dot. Intl. Jol. of scientific research in Physics and Applied Science, 7 (2): 1216.

[22] Qiu Y and Liu Y 2010. Surface chemistry and aspect ratio mediated cellular up take of $\mathrm{Au}$ nanorods. Biomaterials 31: 7606-7609.

[23] Alivisatos P. The use of nanocrystals in biological detection. Nat. Biotechnol. 2004; 22: 47-52. 